\title{
OTIOTOMICS
}

Revista de economía, empresa y sociedad

Dossier "Claves para entender el turismo de hoy"

LOS LÍMITES DEL CRECIMIENTO

\section{H mundo es finito, también para el turismo. Del multiplicador turístico al conficto redistributivo}

\section{Jordi Gascón}

Miembro de la plataforma Foro de Turismo Responsable. Profesor colaborador de la UOC

\section{Frnest Gañada}

Coordinador de Alba Sud

RESUMEN La suposición de que el crecimiento turístico puede ser sostenible está muy difundida en determinados sectores académicos y organismos públicos. La teoría del multiplicador turístico, planteada por Archer y Owen a principios de los setenta, es uno de sus fundamentos. Sin embargo, esta premisa se enfrenta con una realidad cada vez más presente: el planeta y sus recursos son finitos. El desarrollo económico tiene límites naturales. El artículo presenta una visión conflictivista del desarrollo turístico más acorde con esta realidad: el surgimiento de una nueva actividad económica o su crecimiento obliga a reestructurar el uso que se da a los recursos disponibles. Estos recursos no siempre se pueden multiplicar. $Y$ en este proceso, generalmente, unos pierden y otros ganan.

PALABRAS CLAVE turismo; conflicto redistributivo; multiplicador turístico; sostenibilidad

Our world is finite, and so is tourism from tourism multipliers to redistributive conflict

\begin{abstract}
The assumption that tourism growth can be sustainable is widespread in certain academic sectors and public institutions. It is an assumption that is partly founded on the Tourist Multiplier theory, proposed by Archer and Owen in the early 1970s. However, this premise is coming up against an increasingly urgent reality: the finite nature of our planet and its resources. Economic development has natural limits. The article presents a conflictivist view of tourism development that is more in line with this reality: The emergence of new economic activity - or its growth - forces us to restructure our use of the available resources. These resources cannot always be multiplied. And as part of this process, some will lose and others will win.
\end{abstract}

KEYWORDS tourism; redistributive conflict; tourist multiplier; sustainability 


\section{El turismo como estrategia de crecimiento: la Teoría del Multiplicador Turístico}

La teoría del multiplicador turístico fue planteada en los años setenta por Brian H. Archer y Christine B. Owen (1971), y posteriormente ha sido desarrollada y adaptada por numerosos autores (Archer, 1976; Hojo, 2002; Mazumder y otros, 2012). La teoría afirma que el gasto turístico impulsa el desarrollo de los demás sectores económicos. Actuaría como si se tratara de un juego de engranajes en el que la activación de una primera rueda dentada comporta el movimiento de todo el mecanismo. Manuales universitarios y revistas especializadas han difundido este principio sin dudar de su validez (por ejemplo, Prasad Gautam, 2008).

Los estudios sobre el multiplicador turístico se han centrado en el incremento monetario que este sector genera más allá del gasto propiamente turístico. Discernir qué procesos económicos son resultado del desarrollo turístico (Rus y León, 1997) o aplicarlo a casos regionales concretos (Baaijens, Nijkamp y Montfort, 1998; Liu, Var y Timur, 1984; Kauppila y Karjalainen, 2012) se ha llevado una parte de los esfuerzos de análisis economicistas en el ámbito de los estudios turísticos. Esta bibliografía asegura que el eslabonamiento de efectos producidos a partir del consumo turístico tiene lugar a través de dos mecanismos. Por un lado, porque el turismo impulsa la modernización y la construcción de nuevas infraestructuras de transporte, como aeropuertos, puertos, carreteras o redes ferroviarias, que benefician a todos los sectores económicos. Y, por el otro, porque el consumo turístico genera una demanda de bienes y de servicios que convierte a esos sectores en proveedores (Cárdenas García, 2013).

Es innegable que hay casos en que funciona el multiplicador turístico. El turismo gastronómico, por ejemplo, ha demostrado ser una estrategia interesante para revitalizar el sector agrario. Un caso lo encontramos en la Cuina Volcànica de la Garrotxa, propuesta impulsada por un grupo de restaurantes de esta comarca catalana, que se basa en la recuperación y recreación de los usos gastronómicos locales y en la utilización de alimentos producidos en la zona de forma artesanal o por productores campesinos. A partir de estos productos se ha creado una cocina con una personalidad gastronómica reconocida local e internacionalmente, que forma parte de los atractivos turísticos de la comarca junto con sus espacios naturales y sus pueblos de origen medieval. La Cuina Volcànica está ayudando a revalorizar el modelo de producción campesino, proveedor de sus materias primas. Se trata de un modelo agrario caracterizado por producir alimentos de calidad, explotar los agrosistemas de forma sostenible y generar utilidades al ecosistema.

Pero casos como este, aunque valorables, no dejan de ser escasos. Una rápida mirada a destinos turísticos en los que esta actividad se ha convertido en un pilar de la economía descubre que, en muchos casos, el primer sector, lejos de beneficiarse, languidece o ha desaparecido. En otro lugar (Cañada y Gascón, 2016) hemos explicado un caso que es ejemplar: el de la pesca de cabotaje en la costa catalana.

\section{Dudas}

La pesca de cabotaje, de carácter artesanal, generaba empleo a miles de trabajadores y daba vida a los pueblos costeros catalanes. Pero entró en crisis a mediados del siglo pasado, a la par que surgía y se expandía una nueva actividad: el turismo de sol y playa. No fue una casualidad: el desarrollo de la segunda perjudicó a la primera. Así, por ejemplo, la construcción de puertos deportivos (no hay pueblo litoral que no tenga uno) y de otras infraestructuras turísticas y viarias afectó a los ciclos naturales de reposición de la arena costera. Resultado de ello, hoy las playas catalanas desaparecen tras los torrenciales aguaceros otoñales que caracterizan su clima. La necesidad de recuperar la playa antes del siguiente verano para poder iniciar la temporada turística obliga a un bombeo de arena del fondo marino que destroza su ecosistema. Aunque el cada vez más magro sector pesquero y los movimientos ecologistas denuncian esta práctica, los requerimientos del que se ha convertido en sector económico esencial para la economía catalana prevalecen en las decisiones gubernativas. El turismo también disparó los precios del suelo y la vivienda, especialmente de aquellas más cercanas a la primera línea de mar, que ahora se 
destinan a infraestructuras turísticas y segundas residencias. Un proceso de gentrificación que hizo desaparecer los barrios marineros y expulsó a sus habitantes.

No se puede cargar toda la responsabilidad de la desaparición de la pesca en el desarrollo turístico. Otros factores han participado en el proceso, y han tenido un papel igual o más significativo: la contaminación de las aguas por el uso generalizado de agrotóxicos en la agricultura, que acaban en los ríos y acuíferos, y finalmente en el mar, y que afectan también a los ecosistemas pesqueros; la sobrepesca, a medida que se desarrolló la tecnología de detección de cardúmenes y se modernizaron las embarcaciones; la contaminación por vertidos industriales y urbanos. Pero está claro que, en contra de lo que afirma la premisa del multiplicador turístico, el turismo no ha ayudado a revitalizar ese sector, sino más bien todo lo contrario.

Aunque sigue siendo un principio recurrente en estudios académicos y en políticas institucionales, en los últimos años la teoría del multiplicador turístico se ha empezado a poner en duda. Y es que la realidad es testaruda, y ya es imposible obviar los impactos negativos que el desarrollo turístico tiene en las economías y ecosistemas locales. Cada vez son más los estudios que reconocen que el anhelado efecto multiplicador es, más que una realidad automática resultado del desarrollo turístico, un objetivo. Y un objetivo que se enfrenta a numerosos obstáculos (Clavé y González Reverté, 2007). El caso del denominado «enlace» o linkage entre turismo y agricultura, que hemos analizado en otro texto con detalle (Gascón y Ojeda, 2014), es ejemplar.

En los años setenta, con el inicio del boom del turismo internacional, se crearon expectativas sobre las oportunidades que podía generar en la agricultura. Se partía de la premisa de que los destinos turísticos requerirían ingentes cantidades de alimentos para cubrir las necesidades de huéspedes y trabajadores, y que esos suministros se obtendrían con la producción local. Fue Jan Lundgren (1975) quien desarrolló por primera vez esta hipótesis. Lundgren establecía dos posibles escenarios. El primero se daría si el crecimiento hotelero era gradual. En este caso, la demanda también se incrementaría de forma progresiva, y eso daría tiempo a los productores locales para adaptarse a una demanda creciente que requeriría la introducción de innovaciones tecnológicas y la expansión de la frontera agraria. El segundo escenario era la de un crecimiento rápido del destino turístico, basado en la construcción de grandes complejos hoteleros con una fuerte inyección de capital foráneo. En este caso, la demanda de suministros se volvería urgente y sería en grandes volúmenes. En una primera fase, la producción local no podría hacer frente a esta demanda y se dependería de las importaciones. Pero a largo plazo el turismo generaría el estímulo necesario y la producción local se iría adaptando y conquistando espacios en el nuevo mercado. En ambos escenarios, por tanto, el desarrollo turístico acabaría impulsando, sí o sí, el sector agrario de manera natural.

Esta hipótesis se mantuvo con variaciones hasta la década de los noventa (Burns y Holden, 1995; Cox y otros, 1995; Rickard y Carmichael, 1995). Sin embargo, ya en la década antes, diversos estudios dejaban claro que el enlace turismo-agricultura no se estaba produciendo. Por el contrario, los complejos hoteleros preferían importar sus suministros (Bélisle, 1983; Latimer, 1985), cosa que incluso impulsó el dumping: se convirtió en la puerta de entrada de alimentos foráneos que marginaron la producción autóctona de su propio mercado local (Torres, 2003). A principios de siglo, y a partir del trabajo de Rebecca Torres en Quintana Roo (2002, 2003; con Momsen, 2004), se recuperará la hipótesis del enlace turismo-agricultura. Pero ahora con el convencimiento de que el efecto multiplicador no sobreviene de forma espontánea, sino que requiere impulso, planificación y gestión externa. Como decíamos, el multiplicador turístico pasó de ser considerado un proceso automático a convertirse en un objetivo deseable.

\section{Una teorización conflictivista del turismo}

Ya sea de forma natural o inducida, ¿realmente el desarrollo turístico puede beneficiar a los demás sectores económicos locales, y al agrario en particular? Aunque, como hemos explicado, hay casos en que esto sucede, no podemos obviar que el turismo se ha caracterizado más por dañar ecosistemas, malbaratar recursos naturales, mercantilizar expresiones culturales, crear marcos favorables para la corrupción y vulnerar derechos laborales que por lo contrario (Buades, Cañada y Gascón, 2012). 
Cuando se busca la raíz de un conflicto turístico, el análisis suele centrarse en sus causas inmediatas: un resort que enajena agua o tierra a la población local, una disputa entre sectores sociales locales por controlar el nuevo recurso, determinadas políticas municipales que favorecen la gentrificación, etc. Es una aproximación correcta. Pero su reiteración en contextos y geografías diversos nos obliga a buscar un modelo integral del impacto turístico. Creemos que este modelo puede partir de una idea central, que el surgimiento del turismo genera dos tipos de conflicto redistributivo. Por un lado, un conflicto entre sectores económicos que deben competir por unos recursos siempre insuficientes. Por otro, un conflicto entre sectores sociales: entre aquellos que se articulan con éxito con el nuevo sector y los que quedan marginados de sus beneficios.

\subsection{Conflictos redistributivos entre sectores económicos}

El turismo requiere el uso de diferentes recursos (naturales, energéticos, fuerza de trabajo, capital público y privado para la inversión, etc.) que ya están siendo empleados por los sectores económicos prexistentes o por el ecosistema. En contra de lo que implícitamente asegura la teoría del multiplicador turístico, los recursos son finitos. No se pueden reproducir indefinidamente. Por tanto, la aparición del turismo comporta una reestructuración en la asignación de esos recursos. En ocasiones puede que esta reasignación se haga de forma equilibrada, y que tras el reajuste todos los sectores económicos accedan a los recursos necesarios para asegurar su buen funcionamiento. Pero parece predominar una segunda alternativa: el nuevo sector suele sustraer a los ya existentes recursos por encima del mínimo necesario para asegurar su viabilidad. En las zonas rurales donde se establece tiende a decrecer la agricultura, ahogada por el monopolio que el primero hace de recursos como la tierra, el agua, las prioridades de inversión privada, la fuerza de trabajo o los planes de desarrollo gubernamentales (Mowforth y Munt, 2016; Gascón y Ojeda, 2015). El caso de la pesca artesanal que se ha explicado antes es un buen ejemplo.

\subsection{Conflictos redistributivos entre sectores sociales}

Si toda la población participara de forma equitativa en el control y la gestión de los diferentes sectores económicos, entre ellos el turismo, el conflicto anterior tal vez no lo sería tanto: todos se beneficiarían por igual de todos los sectores económicos, estuvieran en crisis o en expansión. Pero esto no suele suceder así. Lo que predomina es una escena en la que el control y la gestión, así como el acceso a los beneficios de cada sector económico, corresponde a sectores de población diferentes. En el mundo rural, esto suele asociarse a una pérdida de control sobre los medios de producción. Un campesino que abandona la actividad agraria para entrar a trabajar en el turismo pasa de una actividad en la que es un especialista y controla los medios de producción (al menos, parcialmente), a otro en el que es mano de obra no cualificada y en cuya gestión no participa. Aunque coyunturalmente pueda obtener unos ingresos atrayentes en la nueva actividad, se ha convertido en mano de obra fácilmente sustituible. Y, por tanto, es desechable si se requiere una reestructuración del sector. Desde los estudios de Richard Butler (1980) sobre el ciclo de vida turístico sabemos que, tarde o temprano, esa reestructuración llegará. Y que esa reestructuración buscará incrementar la competitividad del destino reduciendo los costos de funcionamiento (y, entre ellos, los salarios y condiciones laborales) o mejorando la calidad del servicio (para lo que se hará necesario sustituir la mano de obra por otra cualificada).

\section{Conclusión. La necesidad de considerar la finitud de los recursos}

Una perspectiva que considere el fenómeno desde los conflictos redistributivos entre sectores económicos y sectores sociales tiene la virtud de explicar los impactos del turismo localmente sin olvidar que se trata de un fenómeno global y globalizador. Pero también, y es lo que ahora nos interesa, permite acercarse a la realidad 
turística libre de determinados corsés teóricos como el del multiplicador turístico, que ha servido más de excusa para desviar la mirada de una realidad no deseada que de principio útil para su análisis.

La teoría del multiplicador turístico bebe de una idea de desarrollo que no considera los límites naturales del crecimiento: implícitamente, afirma que se puede crecer de forma indefinida, porque los recursos, por arte de birlibirloque, se pueden reproducir. La mirada conflictivista del fenómeno turístico nos enfrenta con una realidad cada día más evidente: que el mundo y sus recursos son finitos. El surgimiento de un nuevo mercado o de una nueva actividad económica supone tener que restructurar el uso que se da a esos recursos a nuevos objetivos. $\mathrm{Y}$ en este proceso, generalmente, unos pierden y otros ganan.

\section{Referencias bibliográficas}

ARCHER, B.H. (1976). «The anatomy of a multiplier». Regional studies. Vol. 10, n. ${ }^{0}$ 1, págs. 71-77.

ARCHER, B.H.; OWEN, C.B. (1971). «Towards a tourist regional multiplier». Regional Studies. Vol. 5, n. ${ }^{\circ}$ 4, págs. 289-294.

BAAIJENS, S.R.; NIJKAMP, P.; MONTFORT, K. VAN (1998). «Explanatory meta-analysis for the comparison and transfer of regional tourist income multipliers». Regional Studies. Vol. 32, n. ${ }^{\circ}$ 9, págs. 839-849.

BÉLISLE, F.J. (1983). «Tourism and Food Production in the Caribbean». Annals of Tourism Research. Vol. 10, n. ${ }^{\circ}$ 4, págs. 497-513.

BUADES, J.; CAÑADA, E.; GASCÓN, J. (2012). El turismo en el inicio del milenio: Una lectura crítica a tres voces. Madrid: Foro de Turismo Responsable.

BURNS, P.M.; HOLDEN, A. (1995). Tourism: A new Perspective. Londres: Prentice Hall.

BUTLER, R. (1980). «The Concept of a Tourist Area Cycle of Evolution: Implications for Management of Resources». Canadian Geographer. Vol. 24, n. ${ }^{\circ}$, págs. 5-12.

CAÑADA, E.; GASCÓN, J. (2016). «Urbanizar el paisaje: turismo residencial, descampesinización, gentrificación rural. Una introducción». EN: J. GASCÓN; E. CAÑADA (coords.) Turismo residencial y gentrificación rural. Tenerife; Xixón: Pasos Edita; FTR, págs. 5-36.

CÁRDENAS GARCÍA, P. J. (2013). «Análisis de los efectos económicos del turismo». EN: J. I. PULIDO FERNÁNDEZ; P. J. CÁRDENAS GARCÍA (eds.) Estructura económica de los mercados turísticos. Madrid: Síntesis, págs. 43-68.

CLAVÉ, S. A.; GONZÁLEZ REVERTÉ, F. (coords.) (2007). A propósito del turismo: la construcción social del espacio turístico. Barcelona: Editorial UOC.

COX, L. J.; FOX, M.; BOWEN R. L. (1995). «Does tourism destroy agriculture?». Annals of Tourism Research. Vol. 22, n. ${ }^{\circ}$, págs. 210-213.

GASCÓN, J.; OJEDA, D. (2014). Turistas y campesinado: el turismo como vector de cambio de las economías campesinas en la era de la globalización. Madrid; Tenerife: FTR; Pasos Edita.

HOJO, Y. (2002). «The Expansions of the Essays on Tourism Multiplier Model». The Economic Journal of Takasaki City University of Economics. Vol. 45, n. ${ }^{\circ}$ 1, págs. 15-33.

KAUPPILA, P.; KARJALAINEN, T. P. (2012). «A process model to assess the regional economic impacts of fishing tourism: a case study in northern Finland». Fisheries Research. N. ${ }^{\circ} 127$, págs. 88-97.

LATIMER, H. (1985). «Developing-island economies: tourism vs agriculture». Tourism Management. Vol. 6, n. ${ }^{1}$, págs. 32-42.

LIU, J.; VAR, T.; TIMUR, A. (1984). «Tourist income multipliers for Turkey». Tourism Management. Vol. 5, n. 4 , págs. 280-287.

LUNDGREN, J. (1975). «Tourist impact/island entrepreneurship in the Caribbean». EN: R. P. MOMSEN, (ed.). Geographical Analysis for Development in Latin America and the Caribbean. Chapel Hill: CLAG, págs. 12-19.

MAZUMDER, M. N. H. y otros (2012). «Economic Impact of Tourism: A Review of Literatures on Methodologies and Their Uses: 1969-2011». EN: M. KASIMOهLU (ed.). Visions for global tourism industry: creating and sustaining competitive strategies. Rijek: InTech, págs. 269-294. 
MOWFORTH, M.; MUNT, M. (2003). Tourism and Sustainability: Development and New Tourism in the Third World. Londres y Nueva York: Routledge.

PRASAD GAUTAM, B. (2008). Opportunities and Challenges of Tourism Financing. Boca Ratón, Florida: Universal-Publishers.

RICKARD, T.; CARMICHAEL, B. (1995). «Linkages between the Agricultural and Tourism Systems in Sustaining Rural Development in Jamaica». En: I. R. BOWLER y otros (eds.). The sustainability of rural systems: Proceedings, First Meeting of the IGU Study Group on the Sustainable of Rural Systems. Montreal: Universite de Montreal, págs. 316-330.

RUS, G.; LEÓN, C. (1997). «Economía del turismo: Un panorama». Revista de Economía Aplicada. Vol. 15, n. 5 , págs. 71-109.

TORRES, R. (2002). «Toward a better understanding of tourism and agriculture linkages in the Yucatan: tourist food consumption and preferences». Tourism Geographies. Vol. 4, n. 3, págs. 282-306.

TORRES, R. (2003). «Linkages between tourism and agriculture in Mexico». Annals of Tourism Research. Vol. 30, n. ${ }^{\circ}$ 3, págs. 546-566.

TORRES, R.; MOMSEN, J.H. (2004). "Challenges and potential for linking tourism and agriculture to achieve propoor tourism objectives». Progress in Development Studies. Vol. 4, n. 4, págs. 294-318.

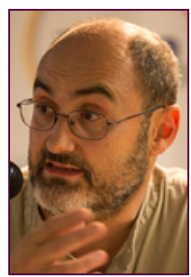

\section{Jordi Gascón}

jgascong@uoc.edu

Miembro de la plataforma Foro de Turismo Responsable. Profesor colaborador de la UOC

Doctor en antropologia social por la Universidad de Barcelona, está especializado en estudios rurales. Sus ámbitos de estudio son los impactos del turismo en el mundo campesino, el turismo como instrumento de la cooperación al desarrollo y las políticas agrarias en America Latina. Desde 1990 realiza investigación en el área andina. Docente de la Universidad de Barcelona y de la Universitat Oberta de Catalunya, es miembro de la plataforma Foro de Turismo Responsable.

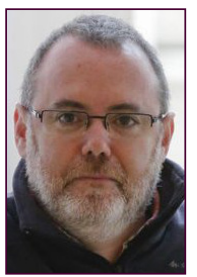

\section{Frnest Gañada}

ernest@albasud.org

\section{Coordinador de Alba Sud}

Máster en Historia Contemporanea por la Universitat Pompeu Fabra, es coordinador de Alba Sud y miembro de la plataforma Foro de Turismo Responsable. Entre 2004 y 2014 residió en Nicaragua, desde donde ha trabajado en el área de Centroamérica, México y Caribe. Su investigación se centra en el turismo comunitario, la conflictividad turística y las condiciones laborales en el sector. 
Los textos publicados en esta revista están sujetas -salvo que se indique el contrario- a una licencia de Reconocimiento 3.0 España de Creative Commons. Podéis copiarlos, distribuirlos, comunicarlos públicamente y hacer obras derivadas siempre que reconozcáis los créditos de las obras (autoría, nombre de la revista, institución editora) de la manera especificada por los autores o por la revista. La licencia completa se puede consultar en http://creativecommons.org/licenses/by/3.0/es/deed.ca.

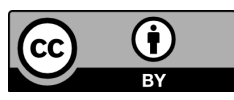

